\begin{tabular}{|c|c|}
\hline 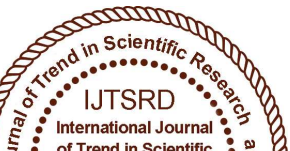 & $\begin{array}{l}\text { International Journal of Trend in Scientific } \\
\text { Research and Development (IJTSRD) }\end{array}$ \\
\hline 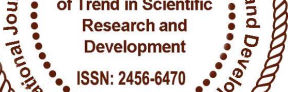 & International Open Access Journal \\
\hline 000 & ISSN No: 2456 - 6470 | www.ijtsrd.com | Volume - 2 | Issue -5 \\
\hline
\end{tabular}

\title{
Voice Recognition Wireless Home Automation System Based on IOT
}

\author{
Kavya K S Math ${ }^{1}$, Dr. Basavaraj Mathpathi ${ }^{2}$ \\ ${ }^{1}$ Student, ${ }^{2}$ H.O.D \\ Appa Institute of Engineering and Technology, \\ Kalaburagi, Karnataka, India
}

\begin{abstract}
In the modern centuries, the Home Computerization scheme that is automation became one of the important challenges using and controlling the things remotely. The burst increase the electronic technology which made an automation to be a part in the day-today life. The remote technology has seen the rise of numerous criterions, mainly in the manufacturing, technical and medical departments.

The wireless home Automation schemes should be executed in present home conditions, devoid of any variations in the structure. The automation hub on acknowledgment of vocal sound instructions and uses a low-power IOT communiqué unit alongside controller. This arrangement is most reasonable for the mature, old and the disabled people particularly those who live alone. The system is proposed to regulate all illuminations i.e. light and electrical procedures in homes based on voice.
\end{abstract}

Keywords: Alexa, Voice, Automation, IOT, Light

\section{PREAMBLE}

\subsection{INTRODUCTION}

The Internet of Things (IOT) is referred to the several physical devices that are connected via Internet around the world in order to collect and share the data over a wireless medium. The IOT can connect the devices, things and people through some communication protocol and some network medium more precisely the wireless medium. The IOT is mainly designed to automate the embedded devices using some sensors to control and monitor the devices and send the data to the cloud storage.

The Wireless Home Automation System is an integrated system to help senior and disabled persons

with a simple to-utilize structure that can be entirely functioned created on voice commands. The compact and development of the system is made in such a way that can be installed, designed and run easily. This kind of arrangement permits to control home hold utilizations from a unified device entity which wirelessly. These usages more often than not need to be well-suited with all additional and with the mechanism entity for most commercially accessible home automation systems.

The enlargement determines the systems that can be incorporated as a solitary versatile component and enables single to remotely regulator lights, air conditioners, security camera's, fans, televisions, doors, CPU systems, sound/visual equipment's etc.

The complete organism is measured using a microphone that is associated to raspberry-pi. This raspberry-pi is having one application so called ALEXA which directs the voice commands in the form of binary to the microcontroller. The base situation entity profits decision according to the voice instruction and directs the instructions to the remote station through Internet. The remote method takes the commands over Wi-Fi enabled internet server and performs the request function.

\subsection{PROBLEM DEFINITION}

To design and develop voice recognition based home automation system through IOT.

\section{LITERATURE SURVEY}

$>$ Kajal Purwar et, al (1)

This paper implemented a Home Automation system using the Raspberry $\mathrm{Pi}$ module and speech to 
command converter. This system can automate the appliances at home. This system may cause some delay as the speech to command converter takes some ample amount of time to convert it to commands.

\section{Gaurav Jadhav et.al (2)}

This paper implemented the Environment Monitoring Scheme using the Raspberry Pi module and Sensors. This system is aimed to monitor and control parameters in a given environment based on the sensor data. The received data is fetch real time and display using the web server.

\section{$>$ Uppugunduru Anil Kumar et.al (3)}

This system was implemented by deploying the sensors in the environment in order to fetch the real time data for the monitoring noise and pollution levels and the data had been send to the cloud. This would be the boom to maintain the pollution at industrial areas.

\section{V.Jyothi et.al (4)}

This paper presents the IOT Based Smart Home System Technologies; the authors have created an Android application the home in order to provide the efficient way of monitoring and controlling electronics appliances of the home environment.

\section{Tasneem Yousuf, et.al (5)}

This paper represents the Internet of Things (IoT) Security: Present Status, Experiments and Countermeasures, this paper elaborates the present status of the IOT in the technology fields the security parameters and all the details.

\section{Snehal K. Dixit et.al (6)}

This paper established the Smart Campus System using IoT in which they developed a Smart campus in which they have enabled the Internet of Things technology and sensor networks to automate the electronic devices in the classical environment to turn into a Smart campus in which the users can get all the information regarding the campus through the internet.

\section{Nagaraj Patil, et.al (7)}

This paper represents a paper on IOT and Raspberry PI Based Environmental Monitoring Application; they developed an open source hardware platform using Raspberry- pi module as the base station and the networking protocol as the XBee. This system measures the environmental conditions using some sensor nodes.

\section{Pragati Ukey et.al (8)}

This paper represents on Development Of Smart Home security system using Raspberry Pi, developed a system for the security purpose for the smart homes Development Of Smart Home security system using Raspberry Pi, the system involves various sensors for controlling different appliances hence ,they have adopted the WI-FI medium for the easy access and maintenance in the remote areas

\section{$>$ Ansiya Jabeen 1 et.al (9)}

This paper represents based on Automatic Classroom Lighting Controller and Energy Saving based on Microcontroller Unit, developed a system which allocates sensor nodes to respective or corresponding light to detect the human body and then accordingly it decides to turn on the power or to keep the lights off thus it helps in saving the energy forms. In the wireless communication where the distance is limited and effective transmission of data

\section{Sarthak Jain et.al (10)}

This paper on Raspberry $\mathrm{Pi}$ based Collaborative Home Automation System over E-mail, they developed a basic system using Raspberry Pi and the E-Mail algorithm and they concluded that its better for huge traffic that's coming in discussion with the previous approaches. They used the Phyton programming and the Raspberry $\mathrm{Pi}$ due to easy implementation and efficiency. Hence this application is said to be user friendly.

\section{PROPOSED DESIGN METHODOLOGY}

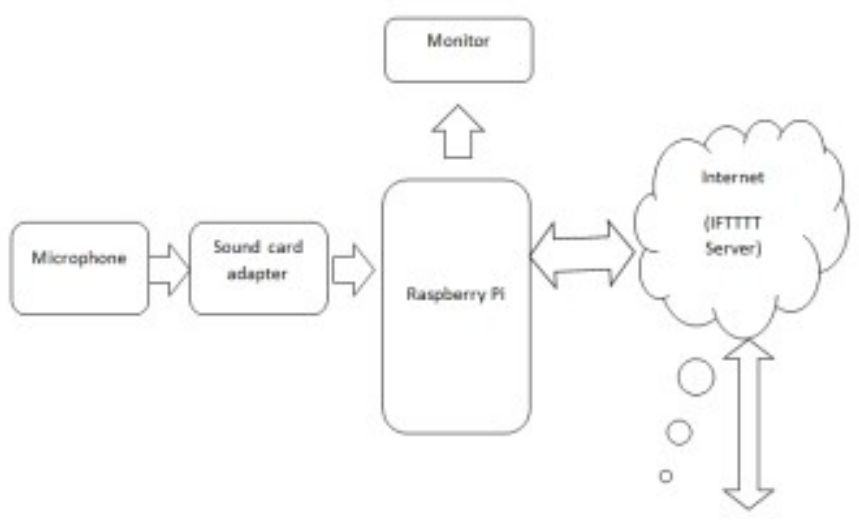

Fig 3.1 Transmitter Unit 


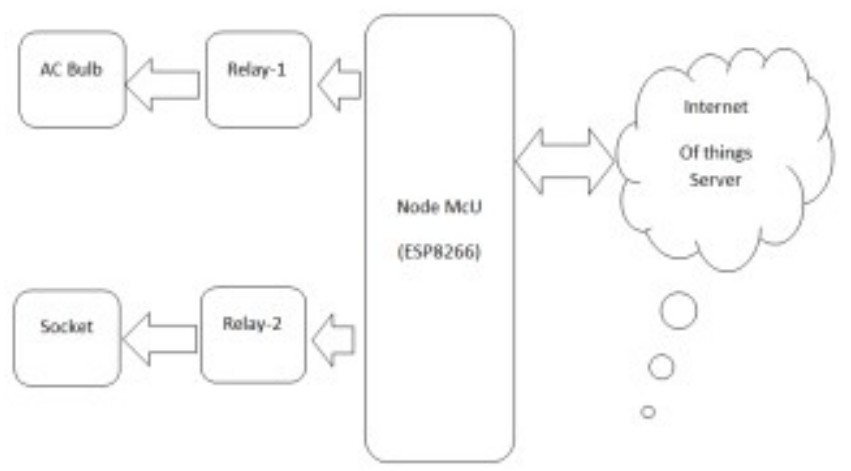

Fig 3.2 Receiver Unit

\subsection{WORKING}

The Smart Home Arrangement is a combined structure to simplify societies with a simple to utilize completely home automatic method. The device functioned centered on speech/talking instructions. The instruction of voice a note is received by raspberry pi and performs the operations. The voice is documented with directives by the Alexa Voice Service (AVS) from Amazon.

The AVS assists users to supplement intellectual speech mechanism to any linked invention that has microphone and speaker. Operators can request Alexa play response interrogations, demand banquet, get news and extra on voice allowable product like the home intercom. Voice is the future and giving a characteristic interface to make an intricate thing straightforward.

\section{Steps of Alexa Voice service:}

Just, Say "Alexa" with a phrase which is assign by the user to turn on and off the devices. It will react permitting to the directives concluded Echo IP Address. For example, if $u$ want to turn on and off the fan by using a command, so $u$ have to say "Alexa turn on fan" or Alexa turn off fan" and so on.

\section{IV.RESULTS AND DISCUSSIONS}

This is the setup of Raspberry Pi for recording voice, the user will give the commands to Alexa through the microphone which is connected to the Raspberry Pi in order to acess the services provided by Amazon Alexa services

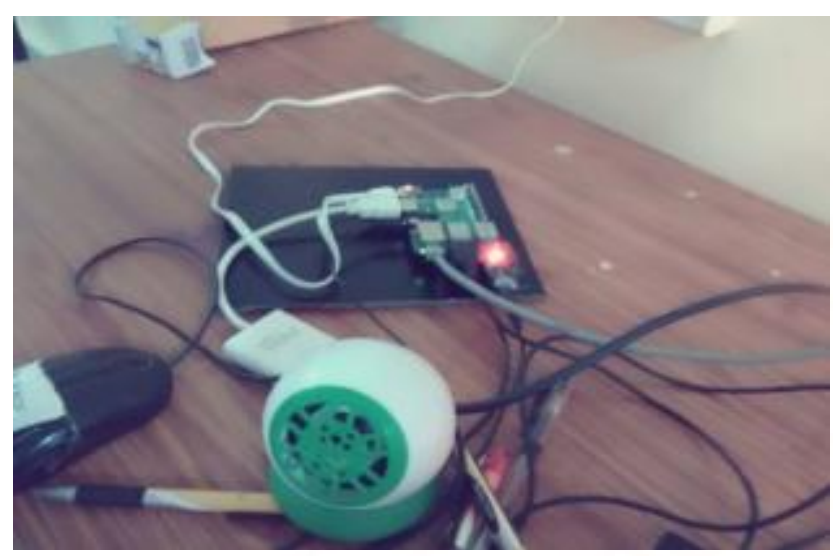

Figure 4.1: Connections for recording Voice commands

The below figure shows the Experimental output model for the system. The bulb is turned on as the output when the relay triggers on upon receiving the voice commands from the Alexa IFTT server.

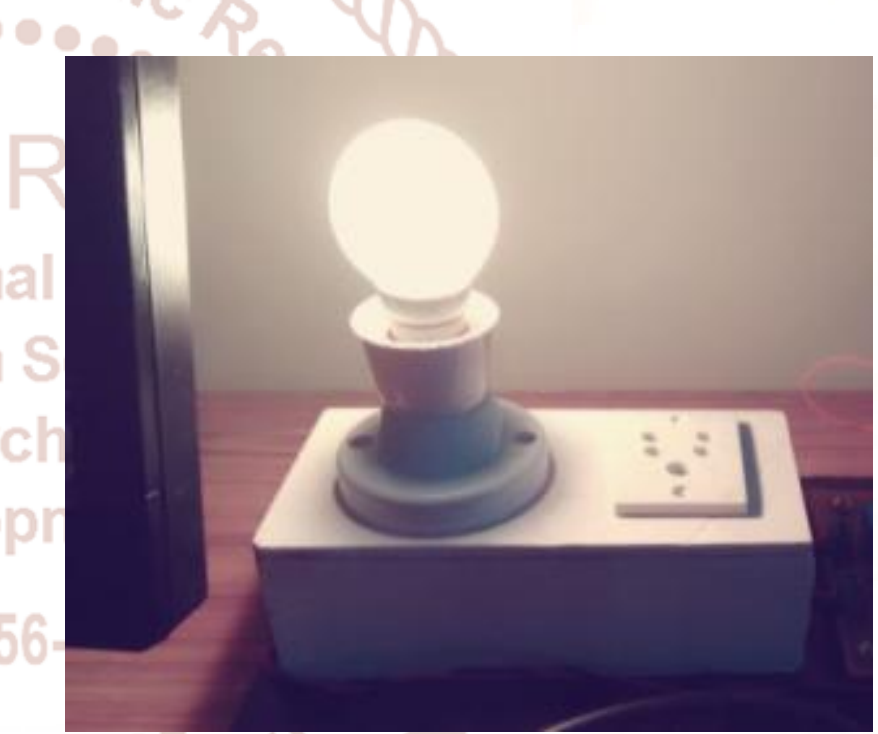

Figure 4.2 : Output Model

\section{CONCLUSION AND FUTURE SCOPE}

The Voice Recognition Wireless Home Automation System is implemented in order to provide many aspects as such improved comfort, cost saving by providing low cost home observing and supervisory method. The system uses the Raspberry Pi which is a open source software that can control many devices programmatically. The of voice/ speech recognition is implemented by the use of Alexa Services provided by the Amazon in order to customize the applets as per the user requirements in order to provide the fully functional and controlled automation through wireless systems.

The future scope of the method is to design in a variety forms. The system further should be secured 
and everybody right to use from every residence place.

\section{REFERENCE}

1. Kajal Purwar, and Ashok Verma 2017, Smart Home Automation System based on IoT through Speech

2. Gaurav Jadhav, Kunal Jadhav, Kavita Nadlamani, 2016 , Environment Monitoring System using Raspberry-Pi

3. Uppugunduru Anil Kumar , G Keerthi, G sumalatha, M. Sushma Reddy, 2017,

IOT BASED NOISE AND AIR POLLUTION MONITORING SYSTEM USING

\section{RASPBERRY PI}

4. V. Jyothi, M. Gopi Krishna, B. Raveendranadh, Debashree Rupalin,2017 IOT Based Smart Home System Technologies
5. Tasneem Yousuf, and 3 others, Internet of Things (IoT) Security: Current Status, Challenges and Countermeasures

6. Snehal K. Dixit, S. M. Kulkarni,2017 Smart Campus System using IoT

7. Nagaraj Patil, Anand K Warad IOT and Raspberry PI Based Environmental Monitoring Application

8. Pragati Ukey +4, Development Of Smart Home security system

9. Ansiya Jabeen1, D. Mahesh Kumar2, Automatic Classroom Lighting Controller Energy Saving based on Microcontroller unit

10. Sarthak Jain, Anant Vaibhav, Lovely Goyal, Raspberry Pi based Interactive Home Automation System through E-mail

11. Dhakad Kunal +4 others, Smart Home Automation using IOT

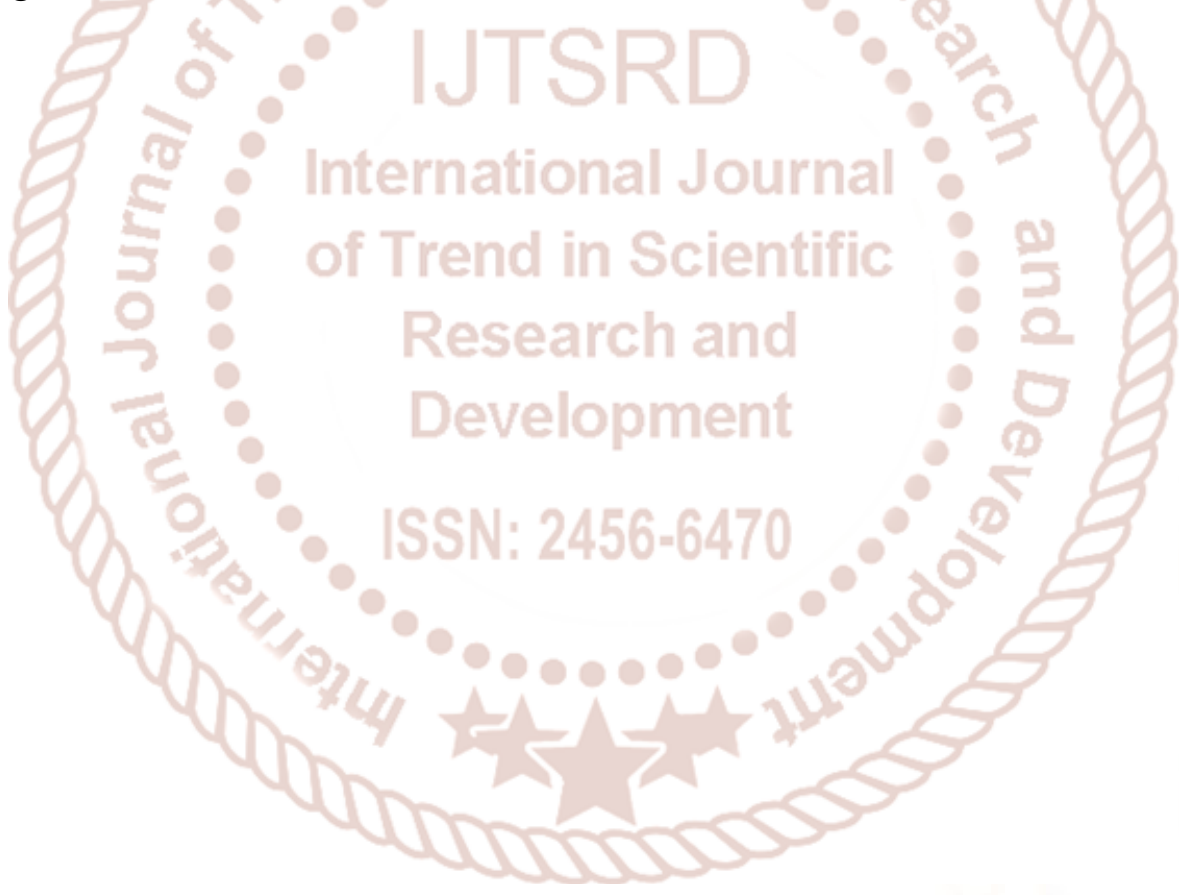

